\title{
On convex lattice polygons
}

\section{P.R. Scott}

Let $\pi$ be a convex lattice polygon with $b$ boundary points and $c(\geq 1)$ interior points. We show that for any given $c$, the number $b$ satisfies $b \leq 2 c+7$, and identify the polygons for which equality holds.

A Zattice polygon $\Pi$ is a simple polygon whose vertices are points of the integral lattice. We let $A=A(I)$ denote the area of $\Pi, b$ (II) the number of lattice points on the boundary of $\Pi$, and $c(I)$ the number of lattice points interior to II .

In 1899 , Pick [2] proved that

$$
A(\Pi)=\frac{1}{2} b(\Pi)+c(\Pi)-1 .
$$

Nosarzewska [1] and more recently Wills [4], have established inequalities relating the area, perimeter, and number of interior points of a convex lattice polygon. It is our purpose here to establish a simple necessary condition for $I$ to be convex.

We set $f(\Pi)=b(\Pi)-2 c(\Pi)$. Using Pick's formula we can obtain alternative expressions for $f(\Pi)$ :

$$
\frac{7}{2} f(\Pi)=b(\Pi)-A(\Pi)-1
$$

and

$$
\frac{7}{2} f(\pi)=A(\Pi)-2 c(\Pi)+1 .
$$

Lattice polygons which can be obtained from one another using integral unimodular transformations or translations are said to be equivalent. The property of convexity, and the quantities $A, b, c$, and $f$ are easily seen to be invariant under equivalence.

Received 12 July 1976. 
The illustrated triangle, $\Delta$ (Figure 1 ) is a lattice polygon of special interest. We observe that

$$
A(\Delta)=\frac{9}{2}, b(\Delta)=9, c(\Delta)=1,
$$

and

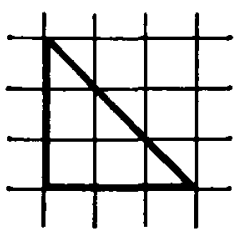

FIGURE 1

$$
f(\Delta)=7 \text {. }
$$

THEOREM. Let $\Pi$ be a convex lattice polygon with at least one interior point. If $\Pi$ is equivalent to $\Delta$, then $f(\Pi)=7$. Otherwise $f(\Pi) \leq 6$.

In the proof of this theorem, we shall make use of the following lemma.

LEMMA. Let $A B, C D$ be segments lying along the $x$-axis, having integral endpoints, and lengths $h, k$ respectively. Let $p$ be a positive integer such that $p>h+k$. Then there exist points $P, R$ on ' $A B, C D$ respectively having integral coordinates, and such that distance $P R$ satisfies

$P R=m+u \quad(m$ a non-negative integer $)$ where $|u| \leq \frac{7}{2}(p-h-k)$.

Proof. Let $A B$ be the segment $[0, h]$, and let $A^{\prime} B^{\prime}$ be the setment $[p, p+h]$ obtained by translating $A B$ through $p$. We may translate $C D$ through integral multiples of $p$ to the position $[t, t+k]$, where $0 \leq t<p$. In fact, we may assume that $h<t<p-k$, else $C D$ overlaps one of the segments $A B, A^{\prime} B^{\prime}$, and we have our result with $u=0$.

Hence we may assume that points $A, B, C, D, A^{\prime}, B^{\prime}$ lie in this order along the $x$-axis. Let $B C=x, D A^{\prime}=y$. Then

$$
\left(B A^{\prime} \Rightarrow\right) p-h=x+k+y \text {; }
$$

that is,

$$
x+y=p-h-k \text {. }
$$

Clearly it is impossible for both $x$ and $y$ to be greater than $\frac{7}{2}(p-h-k)$, and the result follows.

Proof of the theorem. Let $\pi$ meet supporting lines $y=0, y=p$ in segments of length $h, k$ (possibly zero) respectively (Figure 2). 
Since II contains interior points, $p \geq 2$.

Because $\Pi$ is convex, each

horizontal line between $y=0$ and $y=p$ cuts the boundary of $\Pi$ in two points. We deduce that

$$
b(\pi) \leq h+k+2 p .
$$

We now distinguish between several different cases.

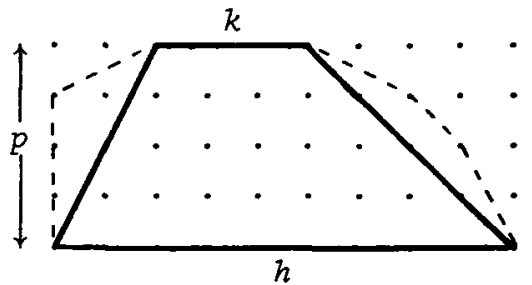

FIGURE 2

Case 1. $p=2$, or $h+k \geq 4$, or $p=h+k=3$. Since $\Pi$ is convex, II contains the convex hull of the two given segments. Hence

$$
A(I) \geq 3 p(h+k)
$$

and

$$
\begin{aligned}
f(\Pi) & =2 b(\Pi)-2 A(\Pi)-2 \\
& \leq 2(h+k+2 p)-p(h+k)-2 \\
& =(h+k-4)(2-p)+6 \\
& \leq 7 .
\end{aligned}
$$

Case 2. $p=3$ and $h+k \leq 2$. Now $b(\Pi) \leq h+k+2 p \leq 8$, and since $c(\Pi) \geq 1, f(\Pi)=b(\Pi)-2 c(\Pi) \leq 6$.

Case 3. $p \geq 4$ and $h+k \leq 3$. Let $\Pi$ meet supporting lines $y=0, y=p$ in points $P, R$ respectively, and supporting lines $x=0, x=p^{\prime}\left(p^{\prime} \geq p\right)$ in points $Q, S$ respectively.

As before, $b(I I) \leq h+k+2 p$. Consider now the effect of transforming $I$ using an integral, unimodular shear having the $x$-axis as invariant line. This transformation leaves $A(\Pi), b(\pi), p, h+k$ unchanged, and preserves the convexity of $\pi$. It may decrease $p^{\prime}$ to a value less than $p$; if this happens, we simply interchange the roles of $p$ and $p^{\prime}$. (There can be at most a finite number of such interchanges, since at each step the positive integer $p+p^{\prime}$ is reduced by at least one.) A further effect of this shear is that all points on the line $y=p$ are translated through some multiple of $p$. Hence by the lemma, it is possible to shear $I$ and choose the points $P, R$ so that the $x$-coordinates of these points differ by $u$, where

$$
0 \leq u \leq \frac{7}{2}(p-h-k) \text {. }
$$




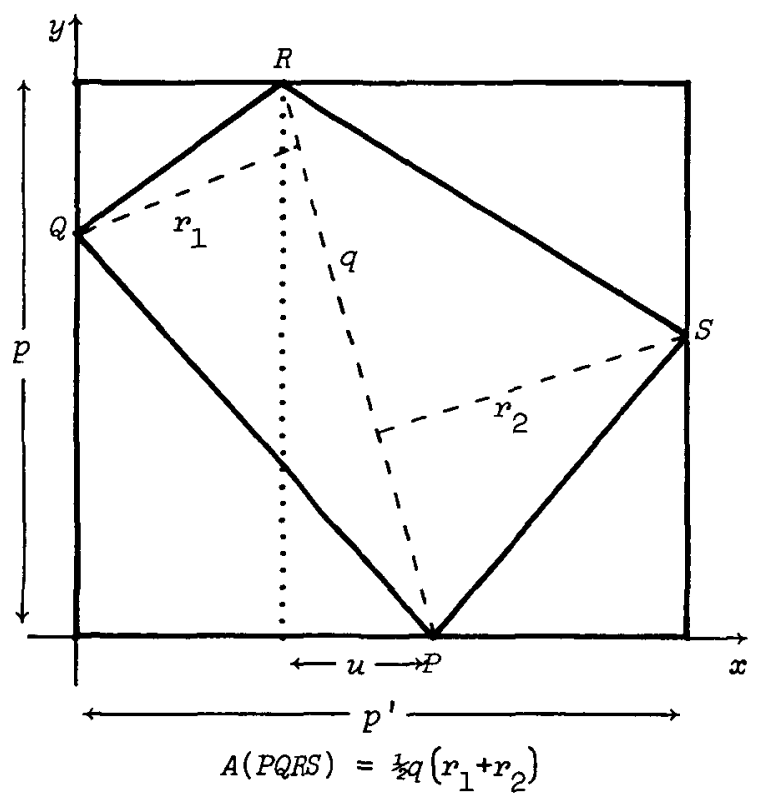

FIGURE 3

Now since $\Pi$ is convex,

$$
\begin{aligned}
A(I I) & \geq A(P Q R S) \\
& =\frac{1}{2} q\left(r_{1}^{+}{ }^{2}\right) \quad \text { (see Figure 3) } \\
& \geq \frac{3}{2} p\left(p^{\prime}-u\right) \\
& \geq \frac{1}{2} p(p-u) \quad \text { since } \quad p^{\prime} \geq p \\
& \geq \frac{3}{4} p(p+h+k),
\end{aligned}
$$

substituting the upper bound for $u$. Hence

$$
\begin{aligned}
f(\pi) & =2 b(\pi)-2 A(\pi)-2 \\
& \leq 2(h+k+2 p)-\frac{3}{2} p(p+h+k)-2 \\
& =\frac{3}{2}(h+k)(4-p)+\frac{3}{2} p(8-p)-2 \\
& \leq 6
\end{aligned}
$$

since $p \geq 4$ and $p(8-p)$ assumes its maximum value of 8 for $p=4$.

Hence in all cases $f(\pi) \leq 7$. For equality here we require $p=3$, $h+k=3, b(\Pi)=9$, and $A(\Pi)=\frac{9}{2}$; it is easily verified that $\Pi$ is equivalent to $\Delta$. The lower value $f(I I)=6$ is attained for a number of lattice polygons $\Pi$, for example lattice rectangles with $p=2$. 
This completes the proof of the theorem.

Finally, we observe that if $c(\pi)=0$, then $f(\pi)$ is unbounded.

This is illustrated by the triangle with vertices $(0,1),(1,1)$, and $(n, 0)$ ( $n$ integral), for which $f(\Pi)=n+1$.

\section{References}

[1] M. Nosarzewska, "Évaluation de la différence entre l'aire d'une région plane convexe et le nombre des points aux coordonnées entières courvertes par elle", colzoq. Math. I (1947/1948), 305-311.

[2] G. Pick, "Geometrisches zur Zahlenlehre", Sitzungsber Lotos Prag. (2) 19 (1900), 311-319. [Quoted from [3], p. 324, and Zentraiblatt über die Fortschritte der Mathematik 31 (1899), p. 215.]

[3] H. Steinhaus, Mathematical snapshots, new edition, revised and enlarged (Oxford University Press, Oxford, London, New York, 1960).

[4] J.M. Wills, "über konvexe Gitterpolygone", Comment. Math. Helv. 48 (1973), 188-194.

Department of Pure Mathematics, University of Adelaide,

Adelaide,

South Australia. 